\title{
Duox mediates ultraviolet injury-induced nociceptive sensitization in Drosophila larvae
}

\author{
Wijeong Jang ${ }^{1}$, Minwoo Baek', Yeon Soo $\mathrm{Han}^{2}$ and Changsoo Kim ${ }^{1 *}$ (D)
}

\begin{abstract}
Background: Nociceptive sensitization is an increase in pain perception in response to stimulus. Following brief irradiation of Drosophila larvae with UV, nociceptive sensitization occurs in class IV multiple dendritic (mdIV) neurons, which are polymodal sensory nociceptors. Diverse signaling pathways have been identified that mediate nociceptive sensitization in malV neurons, including TNF, Hedgehog, BMP, and Tachykinin, yet the underlying mechanisms are not completely understood.

Results: Here we report that duox heterozygous mutant larvae, which have normal basal nociception, exhibit an attenuated hypersensitivity response to heat and mechanical force following UV irradiation. Employing the ppk-Gal4 line, which is exclusively expressed in malV neurons, we further show that silencing duox in mdIV neurons attenuates UV-induced sensitization.

Conclusions: Our findings reveal a novel role for duox in nociceptive sensitization of Drosophila larvae, and will enhance our understanding of the mechanisms underlying this process in Drosophila sensory neurons.
\end{abstract}

Keywords: Duox, ROS, Nociception, Drosophila

\section{Background}

Animals perceive noxious stimuli as pain. Peripheral sensory nociceptive neurons are activated upon nociceptive stimuli and transmit electric signals to central pain pathways, giving rise to pain perception and inducing escape behavior [1]. Nociceptive sensory neurons are 'sensitized' when nearby tissues are damaged, giving rise to pain hypersensitivity, which is manifested as hyperalgesia (pain amplification by painful stimuli) and allodynia (pain creation by non-painful stimuli) [2]. This pain sensitization is beneficial to animal survival since it helps to avoid touching damaged tissues until they are healed. However, in certain pathological conditions, persistent nociceptive sensitization generates chronic pain [3]. The molecular mechanisms underlying nociceptive sensitization are not fully understood.

In Drosophila larvae, class IV multiple dendritic (mdIV) neurons are polymodal nociceptive sensory

\footnotetext{
*Correspondence: changgk2001@hanmail.net

'School of Biological Sciences and Technology, Chonnam National

University, Gwangju 61186, Republic of Korea

Full list of author information is available at the end of the article
}

neurons that induce arborization of dendrites underneath the larval skin $[1,4]$. mdIV neurons acutely respond to diverse noxious stimuli including heat, mechanical force, noxious chemicals and reactive oxygen species (ROS) [1, 5-7]. Diverse ion channels are expressed in mdIV neurons to evoke depolarization in response to corresponding noxious stimuli $[1,5,8-10]$. Next to their acute nociceptive response, mdIV neurons accomplish nociceptive sensitization in response to brief ultravioletinduced tissue damage in the larval skin [11]. Sensitized mdIV neurons give rise to hyperalgesia and allodynia in Drosophila larvae [11]. Like in mammals, tumor necrosis factor (TNF) signaling was shown to operate in mdIV neurons for nociceptive sensitization [11]. Recently, additional signalings including Hedgehog (hh) signaling, Bone Morphogenetic Protein (BMP) signaling and Tachykinin-like signaling have been shown to mediate nociceptive sensitization in mdIV neurons [12-14]. However, the underlying mechanisms are incompletely understood. Here we report the genetic analysis of ROSgenerating Dual Oxidase (Duox) enzymes and find that 
Duox is required to mediate nociceptive sensitization in mdIV neurons.

\section{Results}

To examine whether Duox is involved in pain processing, we undertook a genetic analysis of duox mutants in D. melanogaster. The MiMiC element line MI11825 features an insertion into the 2nd intron of the duox gene (Fig. 1a). Duox [MI11825] homozygotes die as embryos, and are thus not available in the 3rdinstar larval stage for nociception analysis. We therefore used duox [MI11825] heterozygous mutant larvae, in which the transcript level of duox is greatly reduced (Fig. 1b). Duox heterozygous mutant larvae are normal in appearance, larval locomotion, and gentle touch response [15].

\section{Acute nociceptive response is not impaired in duox} heterozygous mutant larvae

Wild-type larvae perceive heat and harsh mechanical force as nociceptive, and thus sensing these stimuli on the skin elicits a characteristic nociceptive response [1] (Additional files 1 and 2). Duox heterozygous mutant larvae exhibit a normal acute nociceptive response to heat and mechanical force, comparable to wild-type larvae (Fig. 1c-d), suggesting that their acute response to nociceptive stimuli is not impaired.
Nociceptive sensitization is impaired in duox heterozygous mutant larvae

Irradiating wild-type larvae briefly ( $5 \mathrm{~s}$ ) with UV induces tissue damage that gives rise to nociceptive sensitization in mdIV neurons [11]. Accordingly, UV-irradiated wildtype larvae exhibited an increased nociceptive response to heat over time (Fig. 2a). Specifically, 20\% of larvae demonstrated nociceptive response to a $40{ }^{\circ} \mathrm{C}$ heat probe, increasing to $40 \%$ of larvae at six hours postirradiation; thus, this nociceptive sensitization is hyperalgesic (amplifies pain). In contrast, homozygous mutant larvae of the transient receptor potential ankyrin 1 (TrpA1) channel, a heat and chemical irritant sensor, exhibited no increased nociceptive response following UV irradiation (Fig. 2b), which is consistent with a published report [11]. Likewise, duox heterozygous mutant larvae exhibited no increased nociceptive response to heat (Fig. 2c), suggesting that duox is required for nociceptive sensitization, and hyperalgesia in particular.

To examine whether duox functions in mdIV neurons, we silenced duox in mdIV neurons employing the pickpocket (ppk)-Gal4 line, which directs expression of Gal4 to mdIV neurons ([16]), and two duox RNAi lines (38,907 and 32,903). When driving expression of duox RNAi with ppkGal4 (ppk-Gal4 > UAS-Duox RNAi 38,907, 32,903), Duox transcript levels were reduced to $64 \%$ for duox RNAi 38,907 and to $78 \%$ for duox RNAi 32,903 (Additional file 3: Figure S1A-B). Importantly, nociceptive sensitization following UV treatment was attenuated for both duox RNAi 38,907

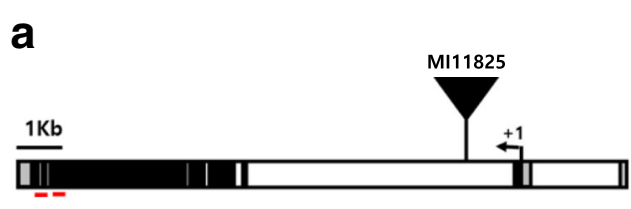

C

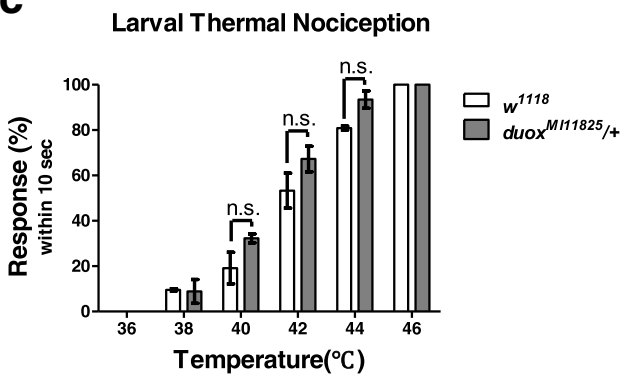

b

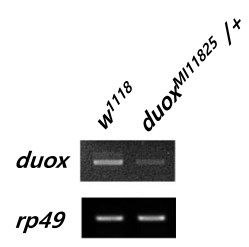

d

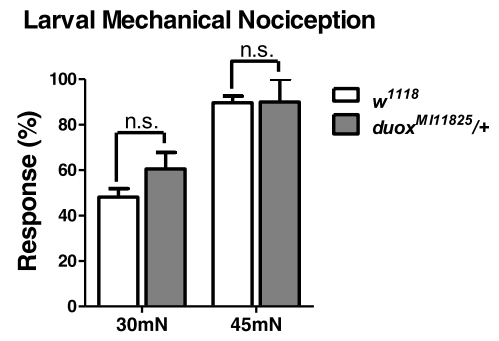

Fig. 1 Basal nociception assay. a The Duox (CG3131) locus. The translation start site is marked with an angled arrow. The MiMiC element (Duox [MI1 1825]) was inserted into the 2nd intron. The UTRs, coding region, and introns are denoted in gray, black, and white, respectively. $\mathbf{b}$ RT-PCR of wild-type (w1118) and Duox [M/11825] heterozygous larvae. Binding sites of the primers used for PCR are indicated by short red lines in (a). The ribosomal protein Rp49 was used as a loading control. c Larval thermal nociception assay. Larval nociceptive response was counted if it occurred within $10 \mathrm{~s}$ of heat exposure ( $n=60$ per time section). $\mathbf{d}$ Larval mechanical nociception. Larval mechanical response to a mechanical force (30 and $45 \mathrm{mN}$ ) was counted ( $n=75$ per each mechanical force). Error bars denote $+/-$ SEM. One-way ANOVA with Tukey post-test was used to analyze the differences. n.s., non-significant 

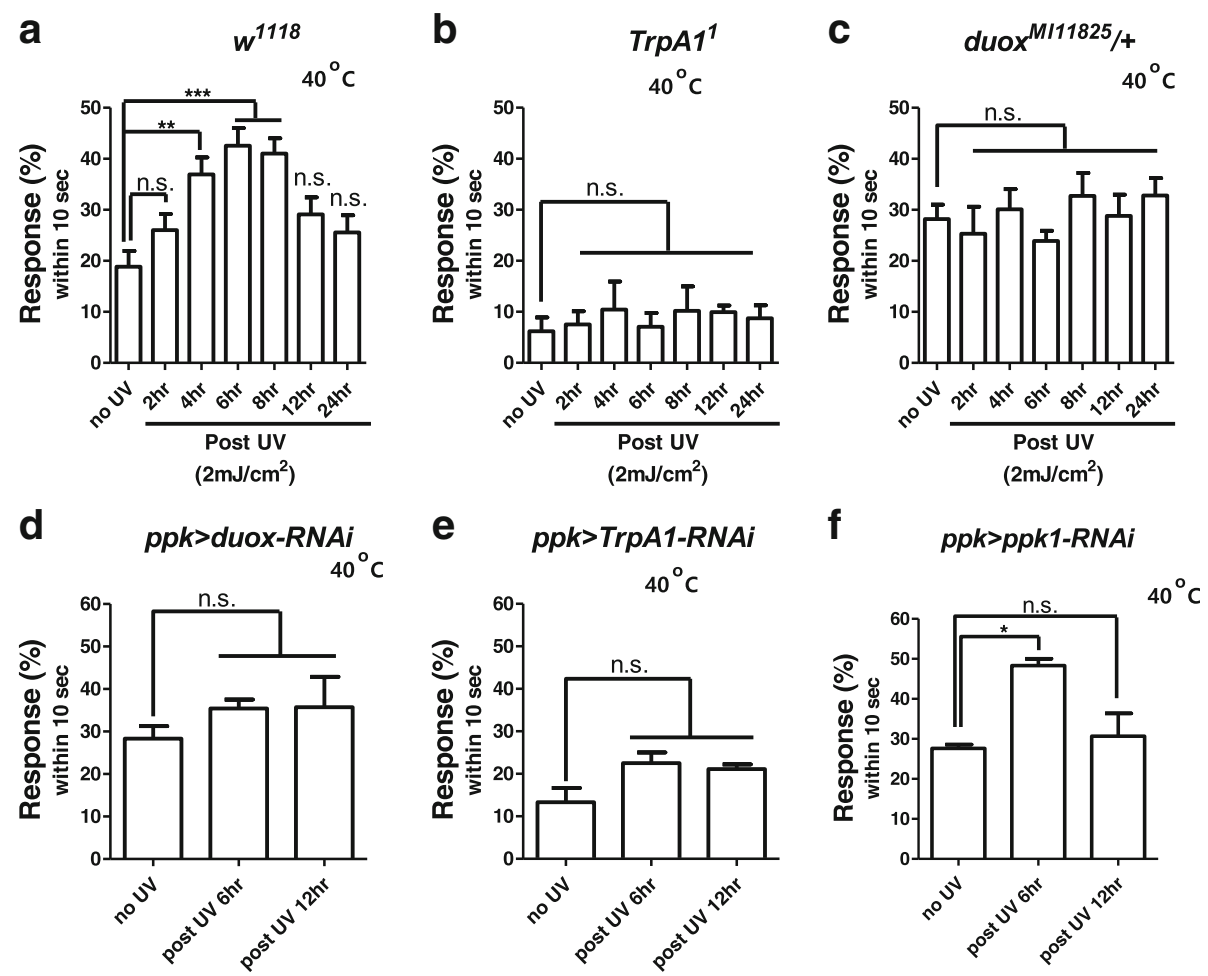

Fig. 2 Thermal nociceptive sensitization assay. Larvae were irradiated with $U V$ at $2 \mathrm{~mJ} / \mathrm{cm}^{2}$. Larval response within $10 \mathrm{~s}$ to heat exposure $\left(40^{\circ} \mathrm{C}\right.$ ) was counted as nociceptive response. a Wild-type lanve ( $n=90$ per time section). b TrpA1 homozygous mutant larvae ( $n=75$ per time section). c Duox heterozygous mutant lavae ( $n=90$ per time section). d Duox silencing in mdlV neurons. Ppk > duox-RNAi denotes ppk-Gal4 > UAS-Duox-RNAi (38907) ( $n=60$ per time section). e TrpA1 silencing in mdlV neurons. Ppk > TRPA1-RNAi denotes ppk-Gal4 > UAS-TrpA1-RNAi ( $n=45$ per time section). f Ppk1 silencing in mdIV neurons. Ppk > ppk1-RNAi denotes ppk-Gal4 > UAS-ppk1-RNAi ( $n=45$ per time section). Error bars denote $+/-$ SEM. One-way ANOVA with Tukey post-test was used to analyze the differences. ${ }^{*},{ }^{* *}$ and ${ }^{* * *}$ indicate $p<0.05,0.01$, and 0.001 respectively. n.S., non-significant

(Fig. 2d) and duox RNAi 32,903 (Additional file 3: Figure S1C). Likewise, silencing of TrpA1 in mdIV neurons (ppk-Gal4 > UAS-TrpA1 RNAi) attenuated nociceptive sensitization following UV treatment (Fig. 2e), consistent with a previous report. However, silencing of $p p k 1$, a mechanosensitive channel, in mdIV neurons (ppk-Gal4>UAS-ppk1 RNAi) did not abrogate heat nociceptive sensitization following UV irradiation (Fig. 2f), suggesting that Ppk1 is not involved in nociceptive sensitization.

It is of note that basal nociception was similar between ppk-Gal4 > UAS-duox (Fig. 2d) and ppk-Gal4 > UAS-ppk1 (Fig. 2f): $\sim 28 \%$ of larvae from either line exhibited nociceptive response against $40{ }^{\circ} \mathrm{C}$ temperatures in the absence of UV irradiation. In contrast, basal nociception was reduced in ppk-Gal4 > UAS-TrpA1 (Fig. 2e), where 12\% of larvae demonstrated nociceptive response against $40{ }^{\circ} \mathrm{C}$ temperatures. These indicate that Duox and Ppk1 are not involved in basal nociception against heat while TrpA1 is, which was previously reported $[17,18]$.

We were additionally curious to learn whether UVirradiated wild-type larvae $\left(w^{1118}\right)$ would exhibit an increased nociceptive response to mechanical force. Similar to the heat response, the increased nociceptive response of wild-type larvae $\left(w^{1118}\right)$ to mechanical force peaks at six hours after UV irradiation (Fig. 3a). In contrast, UVirradiated $\operatorname{Trp} A 1$ homozygous mutant larvae exhibited no increased response to mechanical force (Fig. 3a). Similarly, UV-irradiated duox heterozygous mutant larvae exhibited no increased response to mechanical force (Fig. 3a). In agreement with the mutant analysis, duox and TrpA1 RNAi expression in mdIV neurons (ppk-Gal4 > UAS-Duox RNAi, ppk-Gal4 > UAS-TrpA1 RNAi) attenuated nociceptive sensitization (Fig. 3b-c).

\section{Discussion}

We describe a novel role of duox in nociceptive sensitization in mdIV neurons. Firstly, our data show that duox heterozygous mutant larvae, which exhibit basal nociception, display defective hyperalgesia (pain amplification) to heat and mechanical force following UV irradiation. Secondly, duox silencing in mdIV neurons impairs induced hypersensitivity. Altogether, these genetic studies suggest that Duox is required in mdIV neurons to mediate UV irradiation-derived nociceptive sensitization. 


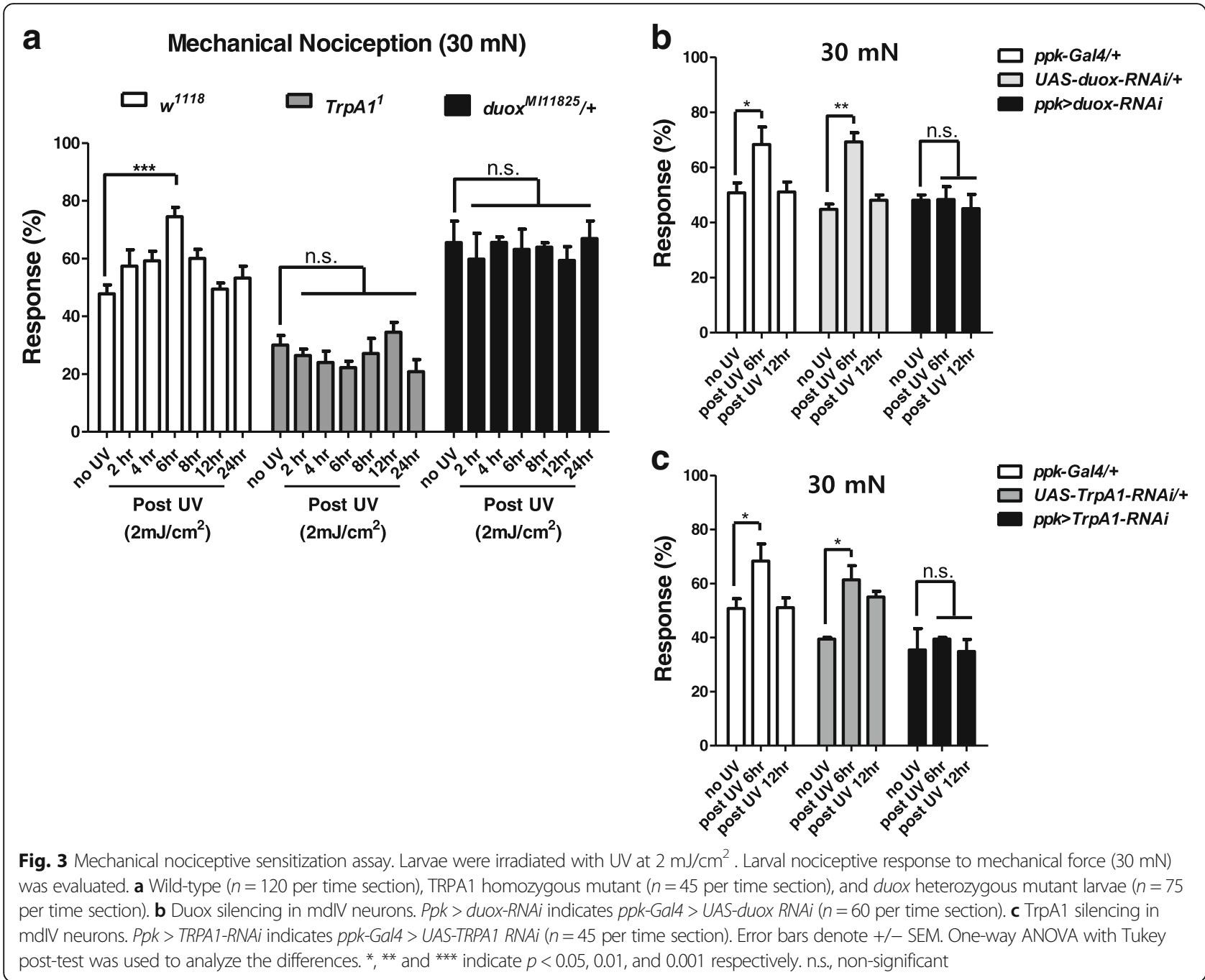

It is of note that $\sim 28 \%$ of larvae expressing either duox or $p p k 1$ RNAi in mdIV neurons ( $p p k>$ Duox-RNAi and $p p k>p p k 1-R N A i$ ) exhibited nociceptive response to $40{ }^{\circ} \mathrm{C}$ heat, as opposed to $\sim 12 \%$ of larvae expressing $\operatorname{TrpA1}$ RNAi. This suggests that silencing of duox or $p p k 1$ in mdIV neurons does not affect basal nociception against $40{ }^{\circ} \mathrm{C}$ heat, while $\operatorname{Trp} A 1$ silencing reduces it. This makes sense in that Duox and Ppk1 are not heat sensors, while TrpA1 is $[5,17,18]$. Notably, duox silencing abrogated heat hypersensitivity while $p p k 1$ silencing did not, highlighting the role of Duox in nociceptive sensitization.

We have shown that basal nociception against heat and harsh mechanical force is not affected by duox reduction, suggesting that mdIV neurons with reduced duox expression retain normal function in sensing nociceptive stimuli and in depolarization. To further confirm this notion, we determined whether structural defects were present in duox heterozygous mutant larvae. We examined the dendrites of duox heterozygotes using $p p k-t d-G F P$ lines that specifically expressed $t d-G F P$ in
mdIV neurons [19]. Confocal images showed that the dendrites of mdIV neurons in duox heterozygous mutant larvae were not reduced in comparison to those of control larvae (Additional file 4: Figure S2); thus, the nociceptive sensitization defects in duox mutants are not due to a reduction of dendrites.

We propose that UV irradiation either directly or indirectly activates Duox expression and/or Duox activation in mdIV neurons. Diverse signaling pathways including TNF, Hedgehog, BMP, and Tachykinin have been shown to mediate UV irradiation-induced nociceptive sensitization in mdIV neurons [11-14]. These signaling pathways could induce the expression and/or activity of Duox [13, 20], and further research should be done to determine whether they do so in mdIV neurons.

The genetic knockdown of heat sensors painless and $\operatorname{Trp} A 1$ abolishes not only basal nociception but also UVinduced nociceptive sensitization [12]. This suggests that Painless and TrpA1 mediate nociceptive sensitization following UV irradiation. Duox is a member of the 
NADPH oxidase family, which produces reactive oxygen species (ROS) in a regulated manner [21]. We speculate that ROS produced by Duox following UV irradiation increase the gating of Painless and TrpA1 through direct oxidation.

\section{Conclusions}

Duox has been shown to catalyze dityrosine cross-links in epithelial cuticles, hormone synthesis, and mucosal immunity in Caenorhabditis elegans, D. melanogaster, and mammals. However, the role of Duox in pain signaling has not been addressed in any animal models. Our data uncover a novel role for Duox in the nociceptive sensitization of sensory nociceptors in Drosophila. Intriguingly, mammalian nociceptors employ a different member of the NADPH oxidase family in nociceptive sensitization [22]. Thus, our findings support the notion that the underlying mechanisms of nociceptive sensitization are evolutionarily conserved from insects to mammals.

\section{Methods \\ Drosophila strains}

Flies were reared on standard yeast/cornmeal agar medium at $25{ }^{\circ} \mathrm{C}$. The ppk-GAL4 (\#32078, \#32079), ppk1-RNAi (\#29571), TrpA1-RNAi (\#31504), duox-RNAi (\#32903, \#38907), duox mutant line (duox $x^{M 111825}$, \#59037) and Ppk-td-GFP (35843) were from Bloomington Drosophila Stock Center.

\section{RT-PCR}

Larvae were collected under $\mathrm{CO}_{2}$ and frozen rapidly in liquid nitrogen. Total RNA from larvae was extracted using TRIzol (MRC) according to the manufacturer's instructions. Reverse transcription (RT) was performed using AccuPower ${ }^{\mathrm{TM}}$ RT Premix (Bioneer K-2041) with $2 \mu \mathrm{g}$ of total RNA in a $20-\mu \mathrm{l}$ reaction. PCRs were performed on an AccuPower PCR Premix (Bioneer K-2016) with duox primers 5 ' - CTGCCCATCGCACAAGCACT$3^{\prime}$ and 5' - CTATCCAAAGTTCTCGAAGT-3' and Rp49 primers 5'-AGATCGTGAAGAAGCGCACC-3' and 5'CACCAGGAACTTCTTGAATCCGG-3'.

\section{UV treatment}

Lightly ice-anesthetized early third-instar larvae were deposited on a $2 \%$ agarose plate and placed in a CL-1000 UV crosslinker (UVP). We used $0 \mathrm{~mJ} / \mathrm{cm}^{2}$ (control) and $2 \mathrm{~mJ} / \mathrm{cm}^{2}$ at a wavelength of $254 \mathrm{~nm}$. After UV treatment, larvae were returned to the rearing medium at $25{ }^{\circ} \mathrm{C}$ before nociceptive sensitivity was assessed at various times after UV exposure.

\section{Behavioral assays}

Larval thermal nociception assays were performed as described previously [1]. Briefly, 3rd instar larvae were placed on $2 \%$ agarose medium in plastic petri dishes, and were laterally touched with a soldering iron with a 0.6 -mm-wide chisel; its temperature was calibrated with a fine thermocouple. The behavioral responses of the larvae were recorded using a digital camera (Kenox, Samsung) and analyzed.

Larval mechanical nociception assays were performed as described earlier [5]. Briefly, 3rd instar larvae were stimulated at $45 \mathrm{mN}$ with a calibrated Sulon monofilament fishing line $(6-1 \mathrm{~b}$ test, diameter $0.23 \mathrm{~mm}$, length $18 \mathrm{~mm}$ ) that was attached to a pipette. Noxious mechanical stimuli were delivered by rapidly depressing the larvae with the fiber on the dorsal side. Each larva was tested only once.

\section{Additional files}

Additional file 1: Video 1. Typical larval behavior upon exposure to non-nociceptive substance. Heat probe, $35^{\circ} \mathrm{C}$. (MP4 $4170 \mathrm{~kb}$ )

Additional file 2: Video 2. Typical larval nociceptive rolling behavior upon heat exposure. Heat probe, $45^{\circ} \mathrm{C}$. (MP4 $4658 \mathrm{~kb}$ )

Additional file 3: Figure S1. A. (left) RT-PCR of 3rd instar larvae from lines Ppk-Gal4/+ (1), Ppk-Gal4 > UAS-duox-RNAi (38907) (2), and Ppk-Gal4 > UAS-DuoxRNAi (32903) (3). The primers used for duox PCR are the same as in Fig. 1. Rp49 was used as a loading control. (Right) Quantification of RT-PCR band areas by Image-J. (NIH). B. The band intensity of duox normalized to that of Rp49, and set to one for Ppk-Gal4/+. C. Larval thermal nociception assay. Rolling within $10 \mathrm{~s}$ of a $40{ }^{\circ} \mathrm{C}$ touch was counted as response ( $n=30$ per time section). Error bars denote +/- SEM. One-way ANOVA with Tukey post-test was used to analyze the differences. ${ }^{*}$ and ${ }^{* * *}$ indicate $p<0.05$ and 0.001 respectively. n.s., non-significant. (PPTX 148 kb)

Additional file 4: Figure S2. Confocal microscopy reveals dendrites of mdIV neurons for Ppk-td-GFP/+ (left) and Ppk-td-GFP/duox [M/11852] larvae (right). These larvae specifically express td-GFP in mdIV neurons. (PPTX $755 \mathrm{~kb}$ )

\section{Acknowledgments}

We thank the Bloomington Drosophila Stock Center, Vienna Drosophila RNAi Center, and Exelixis Drosophila Collection for flies; and the Drosophila Genetic Resource Center for cDNA.

\section{Funding}

This research was supported by Basic Science Research Program through the National Research Foundation of Korea (NRF) funded by the Ministry of Science, ICT and future Planning (Grant No. 2015R1A2A2A01004803).

Availability of data and materials

The datasets are included within the article.

\section{Authors' contributions}

WJ conducted most of the genetic experiments, analyzed the results, and wrote most of the paper. MB conducted initial UV experiments. YSH provided materials. CK conceived the idea and wrote the paper with WJ. All authors read and approved the final manuscript.

Ethics approval and consent to participate

Not applicable

Consent for publication

Not applicable

Competing interests

The authors declare that they have no competing interest with the contents of this article. 


\section{Publisher's Note}

Springer Nature remains neutral with regard to jurisdictional claims in published maps and institutional affiliations.

\section{Author details}

'School of Biological Sciences and Technology, Chonnam National University, Gwangju 61186, Republic of Korea. ${ }^{2}$ Division of Plant Biotechnology, Institute of Environmentally-Friendly Agriculture (IEFA), College of Agriculture and Life Sciences, Chonnam National University, Gwangju 500-757, Republic of Korea.

\section{Received: 21 December 2017 Accepted: 5 March 2018}

Published online: 14 March 2018

\section{References}

1. Tracey WD Jr, Wilson RI, Laurent G, Benzer S. Painless, a Drosophila gene essential for nociception. Cell. 2003;113(2):261-73.

2. Im SH, Galko MJ. Pokes, sunburn, and hot sauce: Drosophila as an emerging model for the biology of nociception. Dev Dyn. 2012;241(1):16-26.

3. Gold MS, Gebhart GF. Nociceptor sensitization in pain pathogenesis. Nat Med. 2010;16(11):1248-57.

4. Hwang RY, Zhong L, Xu Y, Johnson T, Zhang F, Deisseroth K, Tracey WD. Nociceptive neurons protect Drosophila larvae from parasitoid wasps. Curr Biol. 2007;17(24):2105-16.

5. Zhong L, Hwang RY, Tracey WD. Pickpocket is a DEG/ENaC protein required for mechanical nociception in Drosophila larvae. Curr Biol. 2010;20(5):429-34.

6. Xiang Y, Yuan Q, Vogt N, Looger LL, Jan LY, Jan YN. Light-avoidancemediating photoreceptors tile the Drosophila larval body wall. Nature. 2010; 468(7326):921-6.

7. Kim MJ, Ainsley JA, Carder JW, Johnson WA. Hyperoxia-triggered aversion behavior in Drosophila foraging larvae is mediated by sensory detection of hydrogen peroxide. J Neurogenet. 2013;27(4):151-62.

8. Hwang RY, Stearns NA, Tracey WD. The ankyrin repeat domain of the TRPA protein painless is important for thermal nociception but not mechanical nociception. PLoS One. 2012;7(1):e30090.

9. Kim SE, Coste B, Chadha A, Cook B, Patapoutian A. The role of Drosophila piezo in mechanical nociception. Nature. 2012;483(7388):209-12.

10. Jang W, Kim JY, Cui S, Jo J, Lee BC, Lee Y, Kwon KS, Park CS, Kim C. The anoctamin family channel subdued mediates thermal nociception in Drosophila. J Biol Chem. 2015;290(4):2521-8.

11. Babcock DT, Landry C, Galko MJ. Cytokine signaling mediates UV-induced nociceptive sensitization in Drosophila larvae. Curr Biol. 2009;19(10):799-806.

12. Babcock DT, Shi S, Jo J, Shaw M, Gutstein HB, Galko MJ. Hedgehog signaling regulates nociceptive sensitization. Curr Biol. 2011;21(18):1525-33.

13. Im SH, Takle K, Jo J, Babcock DT, Ma Z, Xiang Y, Galko MJ. Tachykinin acts upstream of autocrine hedgehog signaling during nociceptive sensitization in Drosophila. elife. 2015:4:e10735.

14. Follansbee TL, Gjelsvik KJ, Brann CL, McParland AL, Longhurst CA, Galko MJ, Ganter GK. Drosophila nociceptive sensitization requires BMP signaling via the canonical SMAD pathway. J Neurosci. 2017;37(35):8524-33.

15. Kernan M, Cowan D, Zuker C. Genetic dissection of mechanosensory transduction: mechanoreception-defective mutations of Drosophila. Neuron. 1994;12(6):1195-206.

16. Grueber WB, Ye B, Yang $\mathrm{CH}$, Younger $\mathrm{S}$, Borden $\mathrm{K}$, Jan $\mathrm{LY}$, Jan $Y N$. Projections of Drosophila multidendritic neurons in the central nervous system: links with peripheral dendrite morphology. Development. 2007; 134(1):55-64.

17. Neely GG, Keene AC, Duchek P, Chang EC, Wang QP, Aksoy YA, Rosenzweig M, Costigan M, Woolf CJ, Garrity PA, et al. TrpA1 regulates thermal nociception in Drosophila. PLoS One. 2011:6(8):e24343.

18. Zhong L, Bellemer A, Yan H, Ken H, Jessica R, Hwang RY, Pitt GS, Tracey WD. Thermosensory and nonthermosensory isoforms of Drosophila melanogaster TRPA1 reveal heat-sensor domains of a thermoTRP channel. Cell Rep. 2012;1(1):43-55.

19. Han C, Jan LY, Jan YN. Enhancer-driven membrane markers for analysis of nonautonomous mechanisms reveal neuron-glia interactions in Drosophila. Proc Natl Acad Sci U S A. 2011;108(23):9673-8.

20. Kim SH, Lee WJ. Role of DUOX in gut inflammation: lessons from Drosophila model of gut-microbiota interactions. Front Cell Infect Microbiol. 2014;3:116.
21. Ha EM, Lee KA, Park SH, Kim SH, Nam HJ, Lee HY, Kang D, Lee WJ. Regulation of DUOX by the Galphaq-phospholipase Cbeta-Ca2+ pathway in Drosophila gut immunity. Dev Cell. 2009;16(3):386-97.

22. Kallenborn-Gerhardt W, Schroder K, Geisslinger G, Schmidtko A. NOXious signaling in pain processing. Pharmacol Ther. 2013;137(3):309-17.

\section{Submit your next manuscript to BioMed Central and we will help you at every step:}

- We accept pre-submission inquiries

- Our selector tool helps you to find the most relevant journal

- We provide round the clock customer support

- Convenient online submission

- Thorough peer review

- Inclusion in PubMed and all major indexing services

- Maximum visibility for your research

Submit your manuscript at www.biomedcentral.com/submit 